\title{
A NEW IMPROVED THRESHOLD SEGMENTATION METHOD FOR SCANNING IMAGES OF RESERVOIR ROCKS CONSIDERING PORE FRACTAL CHARACTERISTICS
}

\author{
WEI LIN ${ }^{*, \dagger, \ddagger ~ X I Z H E ~ L I, ~},{ }^{*},, \ddagger$ ZHENGMING YANG, ${ }^{*, \dagger, \ddagger}$ LIJUN LIN,$\S$ \\ SHENGCHUN XIONG, ${ }^{\dagger, \ddagger}$ ZHIYUAN WANG, ${ }^{*, \dagger, \ddagger, \|}$ \\ XIANGYANG WANG*,,$+\ddagger$ and QIANHUA XIAO \\ *University of Chinese Academy of Sciences \\ Beijing 100049, P. R. China \\ ${ }^{\dagger}$ Institute of Porous Flow and Fluid Mechanics \\ Chinese Academy of Sciences Langfang \\ Hebei 06500\%, P. R. China \\ ${ }^{\ddagger}$ Research Institute of Petroleum Exploration 83 Development \\ Beijing 100083, P. R. China \\ $\$$ Shandong Agriculture and Engineering University \\ Jinan 250100, P. R. China \\ 'School of Oil and Gas Engineering \\ Chongqing University of Science 85 Technology \\ Chongqing 401331, P. R. China \\ "wangzhiyuan14@mails.ucas.ac.cn
}

Received October 17, 2017

Accepted November 20, 2017

Published February 23, 2018

\footnotetext{
"Corresponding author.

This is an Open Access article published by World Scientific Publishing Company. It is distributed under the terms of the Creative Commons Attribution 4.0 (CC-BY) License. Further distribution of this work is permitted, provided the original work is properly cited.
} 


\begin{abstract}
Based on the basic principle of the porosity method in image segmentation, considering the relationship between the porosity of the rocks and the fractal characteristics of the pore structures, a new improved image segmentation method was proposed, which uses the calculated porosity of the core images as a constraint to obtain the best threshold. The results of comparative analysis show that the porosity method can best segment images theoretically, but the actual segmentation effect is deviated from the real situation. Due to the existence of heterogeneity and isolated pores of cores, the porosity method that takes the experimental porosity of the whole core as the criterion cannot achieve the desired segmentation effect. On the contrary, the new improved method overcomes the shortcomings of the porosity method, and makes a more reasonable binary segmentation for the core grayscale images, which segments images based on the actual porosity of each image by calculated. Moreover, the image segmentation method based on the calculated porosity rather than the measured porosity also greatly saves manpower and material resources, especially for tight rocks.
\end{abstract}

Keywords: Image Segmentation; The Porosity Method; Fractal Theory; Calculated Porosity; Best Threshold.

\section{INTRODUCTION}

The microscopic structure of reservoir rocks is an important factor which affects the reservoir and seepage properties of oil and gas. Therefore, the analysis of the characteristics on the rock microstructure will lay a critical foundation for the study of the micro seepage mechanism of oil and gas.114] With the rapid development of CT imaging technology, the important information which contains the characteristics of the core microstructure can be reflected by the core CT images, CT image has become an important material to analyze the core microstructure. ${ }^{[6]}$ However, the rock skeleton and the pore space in the $\mathrm{CT}$ image can be hard to be clearly distinguished due to the existence of imaging noises and the limitation of instrument precision. So we usually make use of the image binarization algorithm to segment the CT grayscale images, which is recognized as being able to determine the reasonable boundary between the skeletons and the pores. There are many grayscale image binarization algorithms, such as iterative method ${ }^{7]}$ simple statistical method, ${ }^{8}$ Otsu method, ${ }^{9}$ etc. But for these methods, whether it belongs to the global threshold segmentation method $\frac{10[11}{12}$ or the local threshold segmentation method, $\frac{12}{12}$ all do not take into account the important petrophysical information reflected by the image itself, and they only consider the image gray scale features when dealing with images, which will lead to the fact that the segmentation results are theoretically optimal but still far from the actual. Nevertheless, the core porosity is a vital petrophysical parameter and is usually easy to be obtained, which reflects the share of the core pore space, so some scholars have combined this important parameter into the image segmentation, and proposed a threshold segmentation method based on porosity calibration, referred to as the porosity method, 13.14 which can segment the core microstructure images reasonably in theory.

The porosity method can reasonably segment the set of gray values of all the pixels, and the porosity of the obtained black and white images coincide with the experimental porosity. However, it must be noted that, in the case where the core porosity is known, the iterative algorithm is used to select the appropriate gray threshold to segment the CT images, when the porosity of the obtained black and white images are equal to the experimental porosity, the CT image segmentation effect may not be the best. The reason is as follows:

(1) It is a whole core which is usually a standard core with a diameter of $2.5 \mathrm{~cm}$ that is generally used in the laboratory measurement of porosity, however, in order to keep a high resolution, it is a small size core which is usually a core column with a diameter of not more than $5 \mathrm{~mm}$ that is generally selected in CT scan. Since any core has different degrees of heterogeneity, the true porosity of the small size core is not necessarily consistent with the measured porosity of the whole core. 
(2) The porosity of the CT images can only represent the local porosity of the small size core, due to the heterogeneity of the rock, the porosity of the CT images are often different from the porosity of the small size core. Furthermore, on account of the difference of porosity between the whole core and the small size core as stated in the former, it is obvious that the porosity of CT images are not necessarily equal to the porosity of the whole core.

(3) The core porosity measured by the laboratory is the effective porosity, that is, the volume ratio of the connected pores in the core, and the isolated pores in the core can also be displayed in the CT image after CT imaging, so the CT image porosity and the measured porosity are biased.

Moreover, it is convenient to measure the porosity of the conventional rocks under the current laboratory conditions, but for unconventional rocks, such as tight sandstone, shale, etc., the laboratory measurement of porosity usually requires relatively expensive and precision instruments and long measurement cycles.

Fortunately, studies have shown that the pore structures of the rocks have some fractal characteristics, and there is a clear relationship between the fractal dimension (FD) and the porosity of rocks $\frac{15[16}{16}$ Hence, aiming at the problems and shortcomings of the porosity method, combined with the fractal theory, we propose a new improved algorithm of the porosity method to segment the rock scanning images.

\section{THE POROSITY METHOD}

The core is composed of rock skeleton and pore space. In theory, two parts of the core CT image should have very different grayscales. However, due to the constraints of imaging equipment accuracy and other factors, in CT images, although the location of the pores can be roughly distinguished by the naked eye, the difference of the gray level between the pores and the rock skeletons is not very obvious. In addition, the edge of the pores and the skeletons is very fuzzy, which brings great difficulty to the image segmentation. In this case, the accuracy of segmentation is more sensitive to the selection of the threshold.

The porosity of the core is a basic physical quantity that is usually able to be easily and accurately measured by gas expansion method and liquid saturation method and so on, 17-19 under the existing experimental conditions. As the porosity gives the share of the pore space in the rock, it will improve the quality of image segmentation by combining the porosity as the constraint condition to the CT image segmentation. The algorithm to determine the best segmentation threshold by the porosity method is as follows 13114 :

$$
f\left(k^{*}\right)=\min \left\{f(k)=\left|\phi-\frac{\sum_{i=I_{\min }}^{k} p(i)}{\sum_{i=I_{\min }}^{I_{\max }} p(i)}\right|\right\} .
$$

In Eq. (11), $\phi$ is the core experimental porosity, $k$ is the gray threshold, $I_{\max }$ and $I_{\min }$ are the maximum and minimum gray values of the image, respectively. The number of the pixels with the gray value of $i$ is $p(i)$, the pixels with the gray value lower than the threshold of $k$ represent the pore, the rest is on behalf of the skeleton, and $k^{*}$ is the best segmentation threshold.

\section{THE IMPROVED POROSITY METHOD CONSIDERING FRACTAL CHARACTERISTICS}

Fractal geometry, with the ability to describe irregular and disordered objects that traditional Euclidean geometry fails to analyze, was formally proposed by Benoit B. Mandelbrot in 1975 to explore the complexity of objects. $\stackrel{20}{ }$ It was first born in the natural geometry, and shows a broad application prospects in many areas after nearly decades of development.21 26 In 1996, A. M. Vidales obtained the fractal expression for porosity and permeability of fractal porous media. ${ }^{27}$ In 2001, Yu and Li studied the fractal characteristics of porous media, by introducing the fractal scaling law, the porosity of porous media was characterized by fractal, and the formula of fractal dimension based on hard sphere model was put forward! $!^{[5]}$ In 2004 , R. C. K. Wong et al. analyzed the fractal dimension of shale by computer tomography, and obtained the conclusion that shale has fractal characteristics. ${ }^{[28}$ In 2011 , Cai et al. systematically analyzed the study status of imbibition and imbibition coefficient. Based on the fractal characteristics of pore structure of reservoir rocks, the relationship between average actual flow velocity and average linear velocity was derived. And a fractal model of imbibition coefficient for porous media in low permeability reser- 
voirs was established, considering the influence of the original water saturation. ${ }^{29}$ In 2013, Cai et al. established a fractal criterion model of imbibition mechanism, drew the absorption mechanism discrimination diagram and deduced the analytical expression of the structure constant, and found that the smaller the maximum aperture was, the greater the entrance capillary force and the greater the discriminant parameter. $\frac{30}{\mathrm{~A}}$ considerable amount of studies have shown that the pore structure of reservoir rock has typical fractal characteristics, fractal dimension can be used to describe the fractal characteristics of pore distribution. And it is a new method different from the conventional method that fractal geometry theory is used to analyze and study the pore structure characteristics of reservoir rocks, which has been and will continue to be in-depth and widely used in geological analysis. $31+38$

\subsection{Relationship Between Fractal Dimension and Porosity}

According to fractal theory, in the scale-free interval, the cumulative size-distribution of pores should follow the following scaling law 15116 :

$$
N(L \geq \lambda)=\left(\lambda_{\max } / \lambda\right)^{D_{f}},
$$

where $N$ is the cumulative number of pores, $\lambda$ is the pore diameter, $\lambda_{\max }$ is the maximum pore diameter, $D_{f}$ is the fractal dimension for pore size distribution.

Based on Eq. (2), Yu and L 15 derived a relation for fractal dimension for pore volume of saturated/single-phase porous media. This relation analytically relates the fractal dimension $D_{f}$ to porosity and microstructural parameters, $\lambda_{\max }$ and $\lambda_{\min }$, and the relation is given by

$$
\phi_{c}=\left(\frac{\lambda_{\min }}{\lambda_{\max }}\right)^{d_{E}-D_{f}},
$$

where $d_{E}$ is the Euclidean dimension and $d_{E}=2$ and 3 in the two- and three-dimensional spaces, respectively, $\lambda_{\max }$ and $\lambda_{\min }$ are the maximum and minimum pore diameters, $\phi_{c}$ is the porosity.

$\mathrm{Yu}$ and $\mathrm{Li}{ }^{[15]}$ verified the accuracy and validity of Eq. (3) for not only exactly self-similar fractal geometries but also for statistically self-similar fractal geometries (such as random/disordered porous media) by Sierpinski carpet, Sierpinski gasket and textile fabrics. ${ }^{[5]}$ Katz and Thompson ${ }^{23}$ have already presented the experimental evidence from a set of sandstone samples and the results were also in favor of the correlation between Eq. (33) and sandstone indirectly. ${ }^{23}$ Lin et al ${ }^{32}$ successfully applied Eq. (3) to carbonate samples and the calculated porosity of CT images were convincing, which validates the applicability of Eq. (3) on reservoir rocks. .32

\subsection{Calculation of the Parameters $D_{f}, \lambda_{\min }$ and $\lambda_{\max }$}

In this study, $d_{E}=2$. It can be found from Eq. (2) that in the scale-free interval, the cumulative number of pores $N$ and the pore diameter $\lambda$ follow the power-law relation, the expression in the logarithmic coordinates is as follows:

$$
\ln [N(L \geq \lambda)]=-D_{f} \ln \lambda+D_{f} \ln \lambda_{\max } .
$$

Obviously, the cumulative number of pores $N$ and the pore diameter $\lambda$ follow the linear relationship, and the slope value of the line is the fractal dimension $D_{f}$. Using the linear decision method and the automated procedure, the accurate determination of the scale-free interval can be achieved, then the corresponding $D_{f}, \lambda_{\min }$ and $\lambda_{\max }$ are obtained,, 32 and the detailed calculation process can be referred in Ref. 32 .

\subsection{The Improved Porosity Method}

Combined with the fractal theory, a new improved porosity method was proposed and the present method segments images based on the calculated porosity of each image instead of the measured porosity of the whole core. Specifically, first, the actual porosity of each image is calculated by Eq. (3), and then the actual porosity of each image is taken into Eq. (5) to obtain the optimal segmentation threshold, finally, the image is segmented by the optimal threshold:

$$
f\left(k^{*}\right)=\min \left\{f(k)=\left|\phi_{c}-\frac{\sum_{i=I_{\min }}^{k} p(i)}{\sum_{i=I_{\min }}^{I_{\max }} p(i)}\right|\right\} .
$$

\section{CASE ANALYSIS AND DISCUSSION}

This study employed the Xradia XRM-500 CT produced by Xradia company of America, which has the highest resolution of $0.7 \mu \mathrm{m} /$ pixel and is suitable for the establishment of various lithology 3D digital cores. Figure 1 shows a CT grayscale image of a sandstone core collected from a block of Jilin Oilfield, whose scanning resolution is $5 \mu \mathrm{m} /$ pixel 


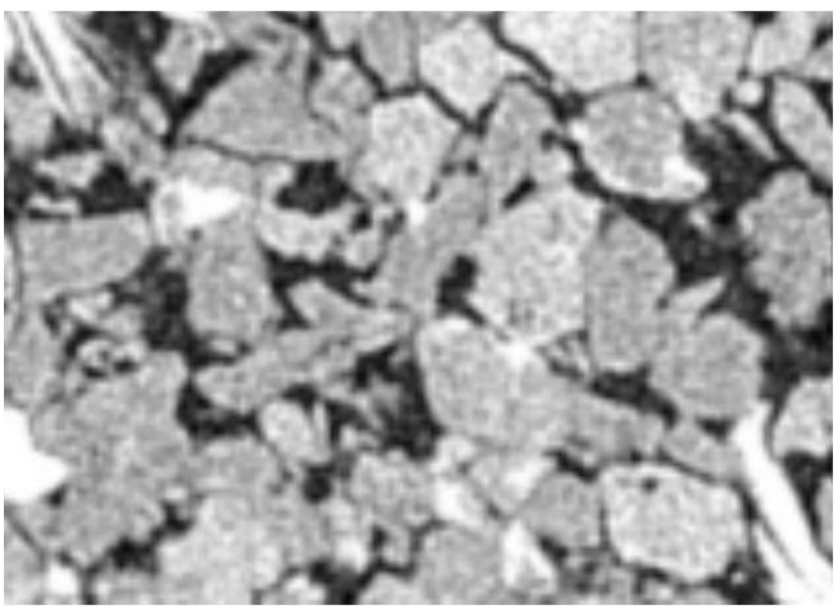

Fig. 1 The CT grayscale image of sandstone core.

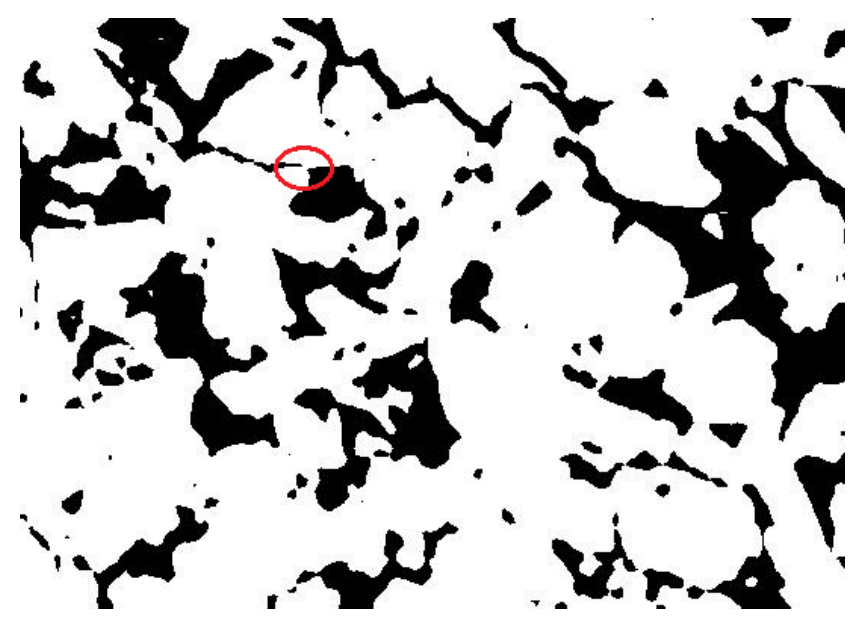

Fig. 2 The black and white image of sandstone core segmented by the porosity method.

and image size is 550 pixels $\times 400$ pixels. The measured porosity of the sandstone core is 0.24 , and the particle size analysis shows that it has a better sorting of diagenetic particles and good heterogeneity. The CT image is segmented by the porosity method and the new method (the improved porosity method), respectively. And the black and white images (the black represent pores, the white represent skeletons) are shown in Figs. 2 and 3, the segmentation thresholds and the porosity of the black and white images are shown in Table 1

As shown in Table 1, it can be seen that the porosity and the segmentation threshold of the image obtained by the new method are slightly larger than those of the porosity method, and the image porosity obtained by the porosity method is consistent with the measured porosity of the whole core, which is determined by the basic principle of the porosity method. However, the image porosity

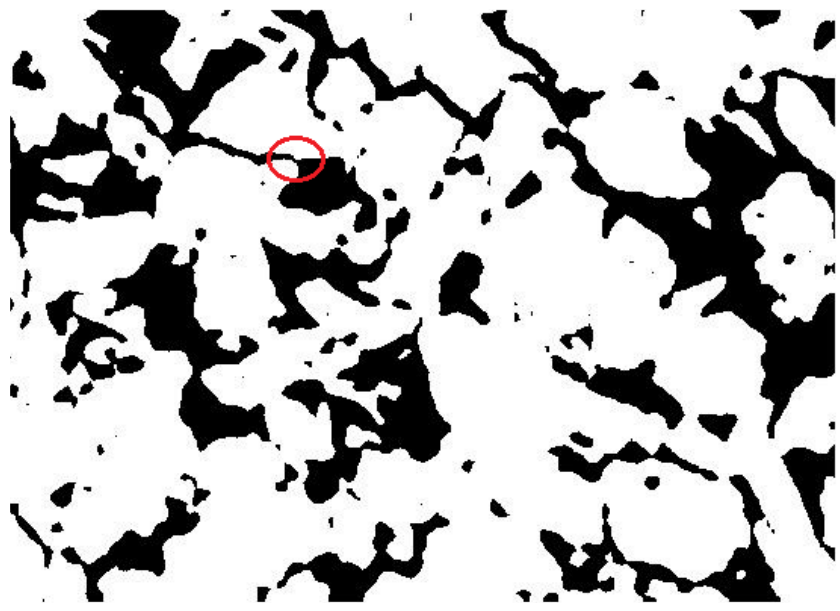

Fig. 3 The black and white image of sandstone core segmented by the new method.

Table 1 Results of Image Segmentation.

\begin{tabular}{lcc}
\hline Parameters & $\begin{array}{c}\text { The Porosity } \\
\text { Method }\end{array}$ & $\begin{array}{c}\text { The New } \\
\text { Method }\end{array}$ \\
\hline Grayscale threshold & 59 & 64 \\
Image porosity & 0.24 & 0.272 \\
\hline
\end{tabular}

of the new method is slightly different from the measured porosity, which should be due to the fact that the core is not completely homogeneous and there are isolated pores. By comparison between Figs. 1 and 2, it is not difficult to find that the proportion of the pores is slightly smaller in the image segmented by the porosity method, which leads to the separation of the connected pores (the marked part of a circle in Fig. 21), so the porosity method needs to be improved. Compared with Figs. 1 and 3, it can be found that the quality of image segmentation by the new method is improved obviously, and the pore size and connectivity (the marked part of a circle in Fig. 31) are more reasonable. The results show that the true porosity of the image is not completely consistent with the measured porosity of the whole core, that is to say, it is not necessary to achieve the best effect with the core experimental porosity as the segmentation criterion. On the contrary, the new method is more reasonable.

In order to verify the effect of the new method on the image processing of unconventional reservoir rocks, we carried out $\mathrm{CT}$ scanning to a tight sandstone core collected from a block of Changqing Oilfield $39 / 40$ The diameter of CT scanning sample is about $2.2 \mathrm{~mm}$, the scanning resolution is $1.6 \mu \mathrm{m}$, and the measured porosity of the sandstone core is 0.086 . At first, the CT scanning images are 
Table 2 Basic Structural Parameters of Digital Cores.

\begin{tabular}{lcc}
\hline Parameters & $\begin{array}{c}\text { The Porosity } \\
\text { Method }\end{array}$ & $\begin{array}{c}\text { The New } \\
\text { Method }\end{array}$ \\
\hline Porosity & 0.086 & 0.091 \\
Connected porosity & 0.071 & 0.085 \\
Pore number & 228 & 246 \\
Throat number & 340 & 413 \\
Average coordination number & 2.67 & 3.02 \\
\hline
\end{tabular}

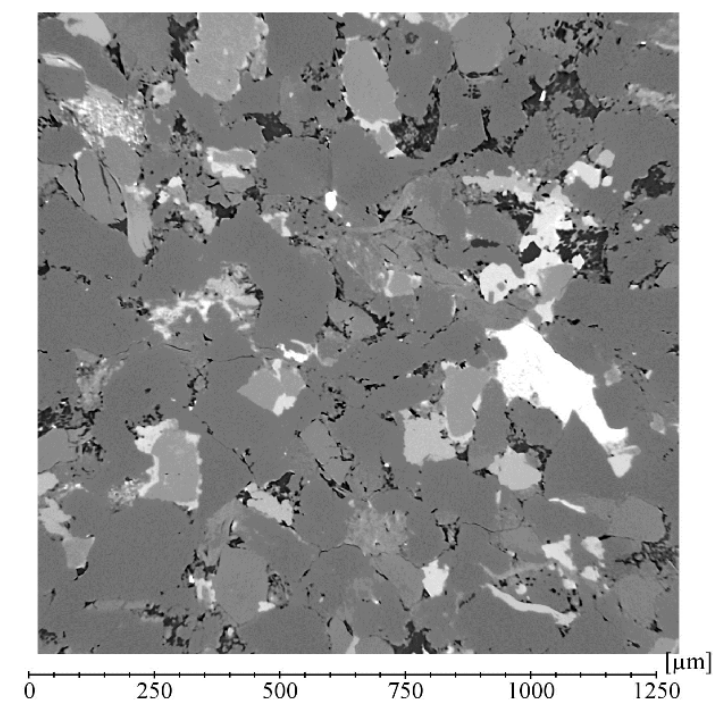

Fig. 4 The CT grayscale image of tight sandstone core.

segmented by the two image segmentation methods, respectively. Then, two kinds of three-dimensional digital cores are constructed by the superposition method, 1143 and the maximum ball algorithm 44,45 is used to extract the digital core pore network models. Finally, using the pore network models as the research platform, the basic structural parameters of the both digital cores can be obtained by counting the pore and throat information in the pore network models (as shown in Table 2). Taking the digital core constructed by the new method as an example, Figs. 4 and 5 display the grayscale image of the core and the black and white image processed by the new method respectively, and the image size is 780 pixels $\times 780$ pixels; Fig. 6 shows the three-dimensional grayscale image of the digital core obtained by the superposition method, whose physical size is $1.25 \mathrm{~mm} \times 1.25 \mathrm{~mm} \times 1.25 \mathrm{~mm}$ and voxel size is $780 \times 780 \times 780$; Figs. 7 and 8 present the skeleton and pore network model of the digital core constructed by the new method, respectively.

According to the data in Table 2, it can be found that the porosity of the digital core established by

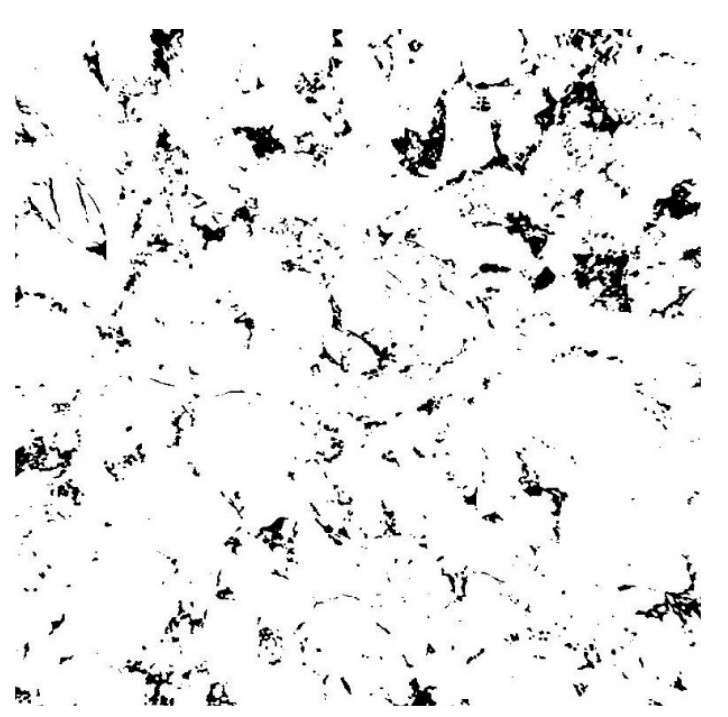

Fig. 5 The black and white image of tight sandstone core.

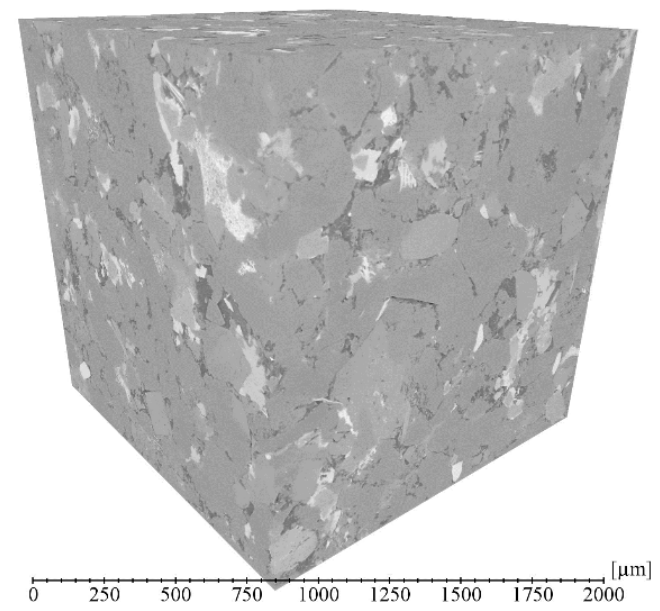

Fig. 6 Three-dimensional grayscale image of the digital core.

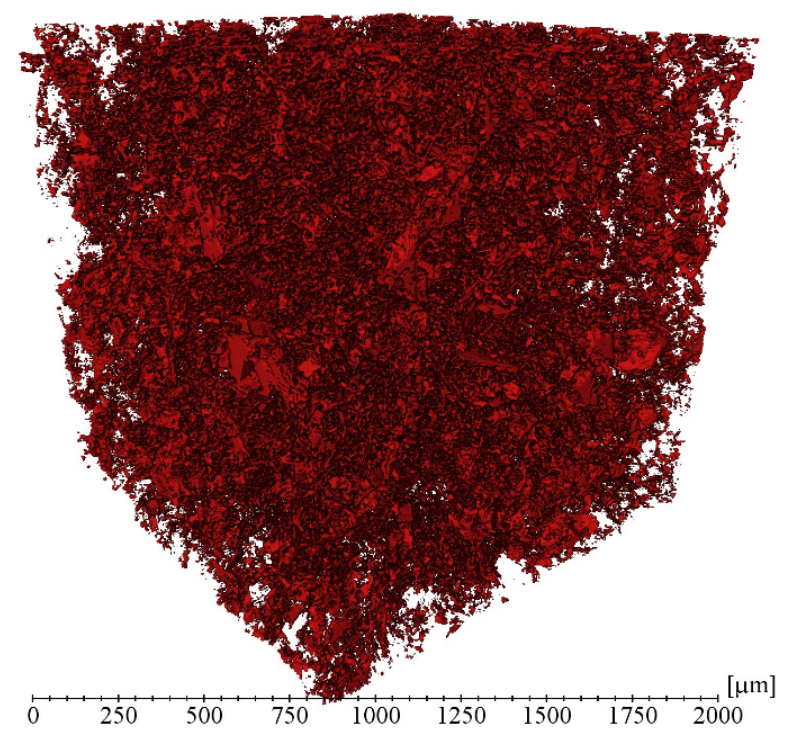

Fig. 7 Three-dimensional skeleton image of the digital core. 


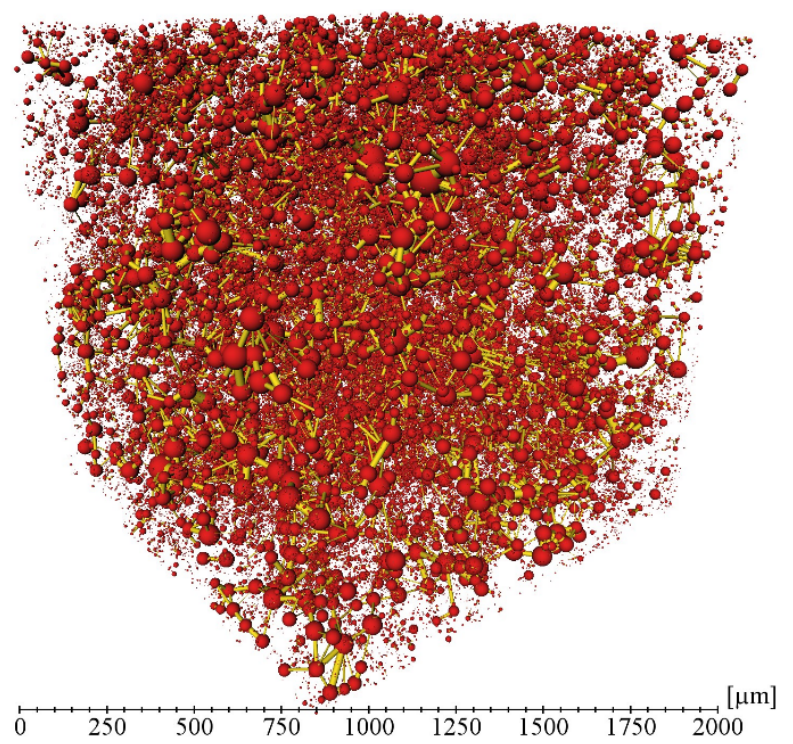

Fig. 8 Pore network model of the digital core.

the new method is slightly higher than the measured porosity of the real core, and the connected porosity is approximately equal to the measured porosity, indicating that there should be $0.6 \%$ isolated pores. However, the porosity of the digital core established by the porosity method is equal to the measured porosity of the real core, and the connected porosity is significantly lower than the measured porosity. The reason is that the measured porosity is the ratio of the connected pore volume to the volume of rock, but the CT images include both the connected pores and the isolated pores, when the CT images are segmented with the measured porosity, some micro pores and throats are mistakenly segmented into skeletons due to the existence of isolated pores, so that the connectivity of the pores is deteriorated and the connected porosity is reduced. The above conclusions are verified by the analysis of pore number, throat number and average coordination number, by comparison, one can find that the pore number of the digital core established by the new method is $7.9 \%$ higher than that of the digital core established by the porosity method, the throat number and average coordination number of the former are $21.5 \%$ and $13.5 \%$ higher than those of the latter, respectively. It fully demonstrates that, due to the existence of isolated pores and the reason that the throat size is usually smaller than the pores, the image segmentation with the porosity method will result in the loss of some minor pores and a considerable amount of throat, which leads to a reduction of coordination number.
The data analysis shows that the new method is reasonable and realistic for the segmentation of the rock pores and skeletons of the tight reservoir rocks, the porosity method ignores the isolated pores and core heterogeneity, and it is not appropriate to use a one-size-fits-all approach to image segmentation and will cause a lot of pores and throats loss.

\section{CONCLUSION}

(1) The threshold segmentation method taken porosity as calibration can reasonably segment the images of reservoir rocks, but it is affected by rock heterogeneity and nonconnected pores. Based on the measured porosity of the whole core, the porosity method adopts a one-sizefits-all approach to image segmentation, which will cause some errors and the microstructure reflected by the binary images different from that of the real core.

(2) The new improved porosity method is the image segmentation method that is reasonable and conforms to the actual images, which takes full account of the existence of rock heterogeneity and isolated pores. In this method, the CT images are segmented by using the calculated porosity of each CT image as calibration, and the microscopic pore structures of the rocks can be preserved to the maximum extent.

(3) The core comparison experiments show that the new improved porosity method overcomes the shortcomings of the porosity method, and makes a more reasonable binary segmentation for the core grayscale images. Moreover, the image segmentation method based on the calculated porosity rather than the measured porosity also greatly saves manpower and material resources, especially for tight rocks.

\section{ACKNOWLEDGMENTS}

We gratefully acknowledge the financial supports from the National Science and Technology Major Project (2017ZX05013-001), the Project of Shandong Province Higher Educational Science and Technology Program (No. J17KB069) and the National Natural Science Foundation of China (51604053).

\section{REFERENCES}

1. F. A. Dullien, Porous Media Fluid Transport and Pore Structure (Academic Press, New York, 1992). 
2. Z. M. Yang et al., Physical and numerical simulation of porous flow pattern in mufti-stage fractured horizontal wells in low permeability/tight oil reservoirs, Acta Petrol. Sin. 35 (2014) 85-92.

3. Z. M. Yang et al., Numerical simulation of the nonlinear flow in ultra-low permeability reservoirs, Petrol. Explor. Dev. 37 (2010) 94-98.

4. X. Z. Li et al., Geological characteristics and development strategies for Cambrian Longwangmiao Formation gas reservoir in Anyue gas field, Sichuan Basin, SW China, Petrol. Explor. Dev. 44 (2017) 428-436.

5. J. T. Fredrich, B. Menendez and T. F. Wong, Imaging the pore structure of geomaterials, Science $\mathbf{2 6 8}$ (1995) 276-279.

6. C. H. Arns and M. A. Knackstedt, Virtual permeametry on microtomographic images, J. Petrol. Sci. Eng. 45 (2004) 41-46.

7. A. Perez and R. C. Gonzalez, An iterative thresholding algorithm for image segmentation, IEEE Trans. Pattern Anal. 9 (1987) 742-751.

8. J. Kittler, J. Illingworth and J. Foglein, Threshold selection based on a simple image statistic, Comput. Vis. Graph. Image Process. 30 (1985) 125-147.

9. N. Otsu, A threshold selection method from graylevel histogram, IEEE Trans. Syst. Man Cybern. 9 (1979) 62-66.

10. J. Kittler and J. Illingworth, Minimum error thresholding, Pattern Recogn. 19 (1986) 41-47.

11. J. N. Kapur, P. K. Sahoo and A. K. C. Wong, A new method for gray-level picture thresholding using the entropy of the histogram, Comput. Vis. Graph. Image Process. 29 (1985) 273-285.

12. S. P. Nie, M. Wang and F. Liu, Image binarization through regional logic computation, Laser Mag. 24 (2003) 48-50.

13. X. C. Zhao, Numerical Rock Construction and Pore Network Extraction (College of Petroleum Engineering in China University of Petroleum, Dongying, 2009).

14. X. J. Liu, H. L. Zhu and L. X. Liang, Digital rock physics of sandstone based on micro-CT technology, Chin. J. Geophys. 57 (2014) 1133-1140 (in Chinese).

15. B. M. Yu and J. H. Li, Some fractal characters of porous media, Fractals 9 (2001) 365-372.

16. B. M. Yu, Analysis of flow in fractal porous media, Appl. Mech. Rev. 61 (2008) 1-19.

17. J. Y. Chen et al., Integrated measurements of permeability, effective porosity, and specific storage of core samples using water as the pore fluid, Int. J. Rock Mech. Min. 79 (2015) 55-62.

18. J. M. Sun et al., Measurement of total porosity for gas shales by gas injection porosimetry (GIP) method, Fuel 186 (2016) 694-707.
19. T. Topór et al., Dual liquid porosimetry: A porosity measurement technique for oil- and gas-bearing shales, Fuel 183 (2016) 537-549.

20. P. Xu, A discussion on fractal models for transport physics of porous media, Fractals 23 (2015) 1530001.

21. J. C. Cai and B. M. Yu, A discussion of the effect of tortuosity on the capillary imbibition in porous media, Transp. Porous Med. 89 (2011) 251-263.

22. J. C. Cai et al., An analytical model for spontaneous imbibition in fractal porous media including gravity, Colloids Surf. A 414 (2012) 228-233.

23. A. J. Katz and A. H. Thompson, Fractal sandstone pores: Implications for conductivity and pore formation, Phys. Rev. Lett. 54 (1985) 1325-1328.

24. B. M. Yu and P. Cheng, A fractal model for permeability of bi-dispersed porous media, Int. J. Heat Mass Transf. 45 (2002) 2983-2993.

25. J. C. Cai et al., Electrical conductivity models in saturated porous media: A review, Earth-Sci. Rev. 171 (2017) 419-433.

26. B. B. Mandelbrot, The Fractal Geometry of Nature (Freeman, San Francisco, 1983).

27. A. M. Vidales and E. N. Miranda, Fractal porous media: Relations between macroscopic properties, Chaos Solitons Fractals 7 (1996) 1365-1369.

28. R. C. K. Wong and M. C. Alfaro, Fractal dimension analysis of shales using X-ray computer tomography, in Int. Workshop on X-Ray CT for Geomaterials Soils, Concrete, Rocks, Kumamoto, Japan, 2004, pp. 239-246.

29. J. C. Cai et al., Fractal analysis on imbibition coefficient in porous media of low permeability reservoir, Geol. Sci. Technol. Inf. 30 (2011) 54-59.

30. J. C. Cai et al., Fractal analysis of spontaneous imbibition mechanism in fractured-porous dual media reservoir, Acta Phys. Sin. 62 (2013) 220-224.

31. W. Wei et al., An electrical conductivity model for fractal porous media, Geophys. Res. Lett. 42 (2015) 4833-4840.

32. W. Lin et al., A method to select representative rock samples for digital core modeling, Fractals 25 (2017) 1740013.

33. M. J. Yun et al., Fractal analysis of power-law fluid in a single capillary, Chin. Phys. Lett. 25 (2008) 616-619.

34. X. H. Tan et al., A simulation method for permeability of porous media based on multiple fractal model, Int. J. Eng. Sci. 95 (2015) 76-84.

35. B. M. Yu, Fractal dimensions for multiphase fractal media, Fractals 14 (2006) 111-118.

36. P. Xu and B. M. Yu, Developing a new form of permeability and Kozeny-Carman constant for homogeneous porous media by means of fractal geometry, Adv. Water Resour. 31 (2008) 74-81. 
37. Y. J. Liu et al., Study of the effect of capillary pressure on permeability, Fractals 15 (2007) 55-62.

38. W. Wei et al., A numerical study on fractal dimensions of current streamlines in two-dimensional and three-dimensional pore fractal models of porous media, Fractals 23 (2015) 1540012.

39. X. Z. Li et al., Efficient development strategies for ultra-deep giant structural gas fields in China, Petrol. Explor. Dev. 45 (2018) 145-148, doi: 10.11698/PED.2018.01.00.

40. X. Z. Li et al., Sedimentary facies of Upper Triassic Xujiahe formation in southwest Sichuan Basin, Nat. Gas Ind. 28 (2008) 54-57.

41. C. H. Arns, The Influence of Morphology on Physical Properties of Reservoir Rocks (The University of New South Wales, Sydney, 2002).
42. J. Coenen, E. Tchouparova and X. Jing, Measurement parameters and resolution aspects of micro $\mathrm{X}$-ray tomography for advanced core analysis, in Proc. Int. Symp. Society of Core Analysts, Abudhabi, 2004, pp. 1-14.

43. W. Lin et al., Construction of dual pore 3-D digital cores with a hybrid method combined with physical experiment method and numerical reconstruction method, Transp. Porous Med. 120 (2017) 227238.

44. A. S. Al-Kharusi and M. J. Blunt, Network extraction from sandstone and carbonate pore space images, J. Petrol. Sci. Eng. 56 (2007) 219-231.

45. H. Dong, Micro-CT Imaging and Pore Network Extraction (Imperial College, London, 2007). 